\title{
Existence of solutions for singular fully nonlinear equations
}

\section{Marcelo Montenegro*}

Universidade Estadual de Campinas, IMECC, Departamento de Matemática, Rua Srgio Buarque de Holanda, 651

CEP 13083-859, Campinas, SP, Brasil

email: msm@ime.unicamp.br

\begin{abstract}
In this note we describe how to approximate some classes of singular equations by nonsingular equations. We obtain a solution to each nonsingular problem and estimates guaranteeing that the limiting function is a solution of the original problem.
\end{abstract}

\section{Introduction}

The following problem was studied in [5]

$$
\left\{\begin{aligned}
-\Delta u & =\chi_{\{u>0\}}\left(-u^{-\beta}+\lambda u^{p}\right) & & \text { in } \Omega \\
u & =0 & & \text { on } \partial \Omega
\end{aligned}\right.
$$

$0<\beta<1$ and $0<p<1$.

Theorem 1.1. There exists a maximal solution for every $\lambda>0$. There is constant $\lambda^{*}>0$ such that for $\lambda>\lambda^{*}$ the maximal solution is positive. And for $\lambda<\lambda^{*}$, the maximal solution vanishes on a set of positive measure.

We solve problem (1) by perturbing the equation as $-\Delta u+\frac{u}{(u+\varepsilon)^{1+\beta}}=$ $\lambda u^{p}$. The solutions $u_{\varepsilon} \searrow u$ pointwise and

$$
\int_{\Omega} u(-\Delta \varphi)+\int_{\{u>0\}} \frac{1}{u^{\beta}} \varphi \leq \lambda \int_{\Omega} u^{p} \varphi
$$

$\forall \varphi \in C^{2}(\bar{\Omega}), \varphi \geq 0, \varphi=0$ on $\partial \Omega$

* Corresponding author.

2000 Mathematics Subject Classification. 34B16, (35J20).

Key words: singular problems, multiple solutions, variational methods. 
There are two approaches to show that $u$ is indeed a solution of (1). Relation (2) tells us that $u$ is a maximal subsolution. We then regularize it and show that $u \in C^{1, \frac{1-\beta}{1+\beta}}$ and indeed solves the problem (1). In doing this, we need to obtain a local estimate $|\nabla u| \leq C u^{\frac{1-\beta}{2}}$ in $\Omega^{\prime} \subset \subset \Omega$. One of the main ingredients is the following Harnack type lemma.

Lemma 1.2. For every ball $B_{r}(p) \subset \Omega$ there are constants $c_{0}, \tau>0$ depending only on $n$ and $\beta$ such that if

$$
f_{\partial B_{r}(p)} u \geq c_{0} r^{\frac{2}{1+\beta}}, \text { then } u(x) \geq \tau f_{\partial B_{r}(p)} u \text { a.e. in } B_{r / 2}(p)
$$

The second approach relies on an estimate for $u_{\varepsilon}$ by the maximum principle, namely $\left|\nabla u_{\varepsilon}\right| \leq C u_{\varepsilon}^{\frac{1-\beta}{2}}$ in $\Omega^{\prime} \subset \subset \Omega$. The idea to obtain such an estimate is to define $v=\frac{\left|\nabla u_{\varepsilon}\right|^{2}}{u_{\varepsilon}^{1-\beta}} \varphi_{1}^{2}$, where $\varphi_{1}$ is the first eigenfunction of the Laplacian with zero boundary condition. The function $v$ has a maximum at $x_{0} \in \Omega$, and then $\Delta v\left(x_{0}\right) \leq 0$. If the estimate is not true, it is possible to take a constant $C>0$ independently of $\varepsilon$ such that $\sup v>C$ and by computation $\Delta v\left(x_{0}\right)>0$, a contradiction. Using the estimate and multiplying the equation by an adequate test function, we let $\varepsilon \rightarrow 0$ in the equation to get a weak solution.

The next problem was studied in $[7]$

$$
\left\{\begin{aligned}
-\Delta u & =\chi_{\{u>0\}}\left(\log u+\lambda u^{p}\right) & & \text { in } \Omega \\
u & =0 & & \text { on } \partial \Omega
\end{aligned}\right.
$$

Both approaches described above work in this case and an analogous result to Theorem 1.1 holds true. The estimate obtained for the maximal subsolution (which is shown to be a solution) is $|\nabla u| \leq C u$ in $\Omega^{\prime} \subset \subset \Omega$ and $u \in C^{1,1}$, a better regularity than the one for (1). This is roughly explained by the fact that $\log u$ is less singular than $-1 / u^{\beta}$. The estimate by maximum principle is $\left|\nabla u_{\varepsilon}\right| \leq C u_{\varepsilon}$ in $\Omega^{\prime} \subset \subset \Omega$.

\section{Fully nonlinear elliptic equations}

We proceed to discuss in more detail the following fully nonlinear problem which was addressed in a work in progress with E. Teixeira [8]. We consider

$$
\left\{\begin{aligned}
F\left(D^{2} u\right) & =G\left(x, u,|\nabla u|^{2}\right) & & \text { in } \quad \Omega \\
u & =f & & \text { on } \partial \Omega
\end{aligned}\right.
$$

with $f \in C^{1, \alpha}(\partial \Omega)$ and $G: \Omega \times \mathbb{R} \times \mathbb{R} \rightarrow \mathbb{R}$ a $C^{1}$ function. Following [3], we define $F: \operatorname{Sym}(d \times d) \rightarrow \mathbb{R}$ and assume $F(0)=0$. The uniform ellipticity 
reads as follows: $\exists \lambda, \Lambda, 0<\lambda \leq \Lambda$ such that

$$
F(\mathcal{M}+\mathcal{N}) \leq F(\mathcal{M})+\Lambda\left\|\mathcal{N}^{+}\right\|-\lambda\left\|\mathcal{N}^{-}\right\|, \forall \mathcal{M}, \mathcal{N} \in \operatorname{Sym}(d \times d) .
$$

In order to state our Lipschitz estimate, let $\phi:(0, \infty) \rightarrow \mathbb{R}$ be such that $\liminf _{s \rightarrow \infty} \phi(s) \geq 0$. We define the asymptotic behavior of $\phi$ passing 0 , $\kappa:(0,1) \stackrel{s \rightarrow \infty}{\rightarrow}(0, \infty)$ by $\kappa(\varepsilon):=\inf \{s: \phi(s)>-\varepsilon\}$.

Theorem 2.1. Let $u \in C^{3}(\Omega)$ be a solution. Define

$$
\sigma(|p|):=\inf _{(x, u)} \frac{D_{u} G\left(x, u,|p|^{2}\right)|p|^{2}-\left|D_{x} G\left(x, u,|p|^{2}\right)\right||p|}{G^{2}\left(x, u,|p|^{2}\right)}
$$

assume $S:=\liminf _{|p| \rightarrow \infty} \sigma(|p|) \geq 0$. Then $\max _{\bar{\Omega}}|\nabla u| \leq C$, where $C$ depends only on $d, \lambda, \Lambda,\|f\|_{C^{1, \alpha}}$ and the asymptotic behavior of $\sigma$ passing 0 .

The proof runs by defining $v=|\nabla u|^{2}$. We compute $D_{i, j} v$ and use the equation. Since $v$ has a maximum at $x_{0} \in \Omega$, we use the asymptotic behavior to conclude the estimate. It is not a proof by contradiction.

Specializing the function $G$ we study the problem

$$
\left\{\begin{aligned}
F\left(D^{2} u\right) & =\beta(u) \Gamma\left(|\nabla u|^{2}\right) & & \text { in } \quad \\
u & =f & & \text { on } \partial \Omega,
\end{aligned}\right.
$$

where $\beta: \mathbb{R} \rightarrow \mathbb{R}$ and $\Gamma:[0, \infty) \rightarrow \mathbb{R}$ are $C^{1, \alpha}$ functions. We have two consequences of Theorem 2.1.

Corollary 2.2. If $\inf _{u} \frac{\beta^{\prime}(u)}{\beta(u)^{2}}>-\infty$ and $\frac{\Gamma(\tau)}{\tau} \rightarrow+\infty$ as $\tau \rightarrow+\infty$, then $\max _{\bar{\Omega}}|\nabla u| \leq C$.

Corollary 2.3. If $\beta$ is nondecreasing, $|\beta|+\left|\beta^{\prime}\right|>0$ and $\liminf _{\tau \rightarrow \infty} \Gamma(\tau)>0$, then $\max _{\bar{\Omega}}|\nabla u| \leq C$.

Definition: $u$ is a viscosity subsolution in $\Omega$ if $F\left(D^{2} u\right) \geq g$ in the viscosity sense in $\Omega$ if, that is, for every $x_{0} \in \Omega, V_{x_{0}}$ neighborhood, $\varphi \in C^{2}\left(V_{x_{0}}\right)$, $u \leq \varphi$ in $V_{x_{0}}, u\left(x_{0}\right)=\varphi\left(x_{0}\right)$, then $F\left(D^{2} \varphi\left(x_{0}\right)\right) \geq g\left(x_{0}\right)$.

Definition: $u$ is a viscosity supersolution in $\Omega$ if $F\left(D^{2} u\right) \leq g$ in the viscosity sense in $\Omega$ if, that is, for every $x_{0} \in \Omega, V_{x_{0}}$ neighborhood, $\varphi \in C^{2}\left(V_{x_{0}}\right)$, $u \geq \varphi$ in $V_{x_{0}}, u\left(x_{0}\right)=\varphi\left(x_{0}\right)$, then $F\left(D^{2} \varphi\left(x_{0}\right)\right) \leq g\left(x_{0}\right)$. It is possible to refrase this definition with $\varphi$ being a quadratic function.

A viscosity solution is a continuous function $u$ which is a subsolution and a supersolution. 
Definition: a continuous function $u$ satisfies $F\left(D^{2} u\right)=+\infty$ at a point $X_{0}$ if $u$ cannot be touched from above by a smooth function at $X_{0}$.

The idea behind the above definition relies in the fact that if $u(x)=|x|$, then $\Delta u=(n-1) /|x|$ in the distributional sense. We could say that $\Delta u(0)=+\infty$. In the light of the viscosity theory, given an arbitrary positive number $K, P_{K}(x)=\frac{K}{2 n}|X|^{2}$ touches $u$ at 0 from below. Indeed, $P(0)=u(0)$ and in $0<|x|<\frac{1}{K}$, we have $u(x)>P_{K}(x)$. Thus, " $\Delta u(0) \geq$ $K$ " for every $K$.

Definition: a continuous function $u$ is a viscosity solution in the topological sense if it satisfies $F\left(D^{2} u\right)=+\infty$ at a point $X_{0}$.

The approach to solve (3) is by considering again a perturbed problem

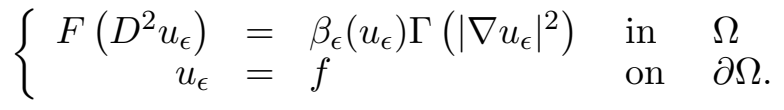

Using Corollary 2.2 we derive existence of a Lipschitz viscosity solution in the topological sense for

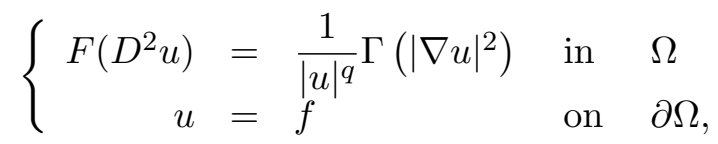

with $q \geq 1, \Gamma \geq 0, \Gamma$ superlinear and $F$ concave. In this case $\beta_{\epsilon}(u)=1 / u^{q}$ for $u>\varepsilon$ and $\beta_{\epsilon}(u)=\varepsilon$ for $u<-\varepsilon$. Between $-\varepsilon$ and $\varepsilon, \beta_{\epsilon}(u)$ is a fourth order polinomial. Since $\beta_{\epsilon}(u)$ is not monotone, Perron's method should be adapted by adding a term $k u$ in both sides of the equation. This gives a solution $u_{\varepsilon}$ to (4). The estimate of Theorem 2.1 allows us to let $u_{\varepsilon} \rightarrow u$, thus obtaining a viscosity solution of (5).

Another existence of viscosity solution result can be obtained using Corollary 2.3 for the problem

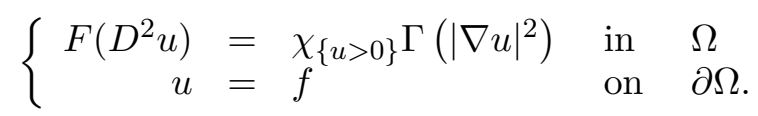

In this case $\beta_{\varepsilon}$ is defined as follows. Let $\rho$ be a smooth function supported in $[0,1], \rho>0$ in $(0,1)$ and normalized as to $\int_{\mathbb{R}} \rho=1$. We define

$$
\beta_{\epsilon}(s):=\frac{1}{2} \int_{0}^{s / \epsilon} \rho(\tau) d \tau-\frac{1}{2} \int_{0}^{-s / \epsilon} \rho(\tau) d \tau+\frac{1}{2}+\epsilon,
$$

which satisfy the assumptions of Corollary 2.3.

Equations similar to (5) and (6) have been treated in $[4,6]$. The solutions of the equations may exhibit a free boundary, whose regularity can be studied with techniques from [1]. 
Another problem that could be treated with our techniques is

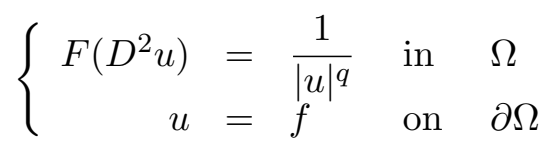

where $0<q<1$ and $F$ is concave. There is a viscosity solution in the pointwise topological sense. Moreover, $u \in C^{1, \frac{1-q}{1+q}}$, the regularity of the first problem (1).

In the proof we use a version of Theorem 2.1 and ideas from the proof of existence of solution to problem (5). Here $\beta_{\epsilon}$ is the same used to solve (5) and is not monotone.

\section{Examples and comparison to our results}

In problem (5) we have shown existence of solution to

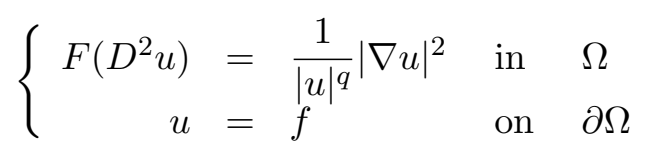

if $q \geq 1$. There is a result in [2] saying that

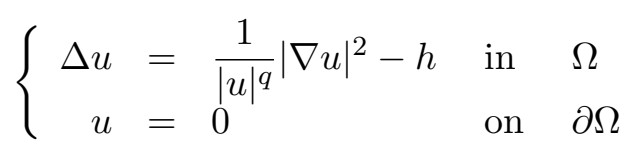

has a $\geq 0, \not \equiv 0$ solution if and only if $q \leq 2$, provided $q>0$ and $h$ is smooth and positive at every compact subset of $\Omega$.

By problem (7) we know that

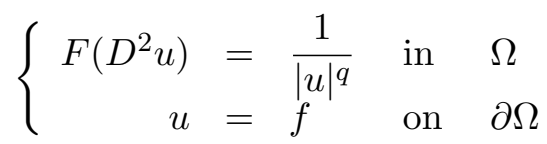

has a solution if $0<q<1$, remember $f \geq 0$. The first problem (1)

$$
\left\{\begin{aligned}
\Delta u & =\frac{1}{|u|^{q}} & & \text { in } & & \Omega \\
u & =0 & & \text { on } & & \partial \Omega
\end{aligned}\right.
$$

has no positive solution if $0<q<1$. Notice that

$$
\left\{\begin{aligned}
-\Delta u & =\chi_{\{u>0\}}\left(-u^{-q}+\lambda\right) & & \text { in } \Omega \\
u & =0 & & \text { on } \partial \Omega
\end{aligned}\right.
$$

has no solution if $q \geq 1$. 
But by problem (6)

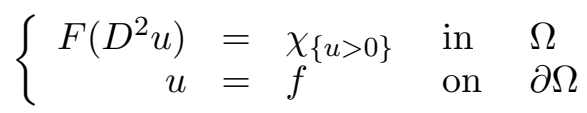

has a solution.

Acknowledgement. The author would like to thank the organizers of the IST-IME meeting for their kind invitation. He was partially supported by CNPq.

\section{References}

[1] H. W. Alt and L. Caffarelli, Existence and regularity for a minimum problem with free boundary, J. Reine Angew. Math. 325 (1981), 105-144.

[2] D. Arcoya, J. Carmona, T. Leonori, P. Martnez-Aparicio, L. Orsina, F. Petitta, Existence and nonexistence of solutions for singular quadratic quasilinear equations, J. Differential Equations 246 (2009), 4006-4042.

[3] X. Cabré and L. Caffarelli, Fully nonlinear elliptic equations. American Mathematical Society Colloquium Publications, 43. American Mathematical Society, Providence, RI, 1995.

[4] M. Crandall, P. Rabinowitz and L. Tartar, On a Dirichlet problem with a singular nonlinearity, Comm. Partial Differential Equations 2 (1977), 193-222.

[5] J. Dávila and M. Montenegro, Positive versus free boundary solutions to a singular elliptic equation, J. Anal. Math. 90 (2003), 303-335.

[6] A. C. Lazer and P. J. McKenna, On a singular nonlinear elliptic boundary-value problem, Proc. Amer. Math. Soc. 111 (1991), 721-730.

[7] M. Montenegro and O. Queiroz, Existence and regularity to an elliptic equation with logarithmic nonlinearity, J. Differential Equations 246 (2009), 482-511.

[8] M. Montenegro and E. Teixeira, Gradient estimates for viscosity solutions of singular fully non-linear elliptic equations

[9] J. Shi and M. Yao, On a singular nonlinear semilinear elliptic problem, Proc. Roy. Soc. Edinburgh Sect. A 128 (1998), 1389-1401. 